\title{
EDITORIAL
}

\section{Heart failure: a global disease requiring a global response}

\section{J E Sanderson, T-f Tse}

Heart 2003;89:585-586

\section{Economic globalisation is causing the incidence and prevalence of heart failure to increase at an alarming rate around the world}

$T$ he heart is arguably the organ that suffers most obviously from increasing economic globalisation. Although mortality from coronary artery and cerebrovascular diseases has been declining in western Europe and North America since the mid to late 1970s, this is not the case elsewhere in the world. ${ }^{1}$ Increasing industrialisation and urbanisation, while raising living standards and increasing lifespan for many, is also accompanied by a change of diet (increased calories, fat and salt), cigarette smoking, lack of exercise, and obesity. ${ }^{2}$ Diabetes, hypertension, and coronary artery disease are the initial consequences and heart failure follows, which is the end product of myocardial damage caused by these disease processes alone or more usually in combination.

In nearly all regions of the world heart failure is both common and on the rise. ${ }^{34}$ In Hong Kong, based on admissions to a teaching hospital serving a large community, the incidence rate is $3-3.8 / 1000 /$ year rising to $20 / 1000 /$ year in women over the age of 85 years. $^{5}$ This is comparable to rates in western countries that range from 1 to 5 cases/1000/year. ${ }^{3}$ And in Hong Kong there has been a $10 \%$ annual increase in hospital admissions over the past five years (unpublished data). In Africa, at least $3-7 \%$ of all hospital admissions are caused by heart failure. ${ }^{6}$ In South America cardiovascular disease is now the leading cause of death, and the prevalence rate of heart failure is about $4 \%$ in those older than 65 years. ${ }^{4}$ Similar prevalence rates were found in an Arab population. ${ }^{7}$

\section{AETIOLOGY}

The cause of heart failure varies somewhat around the world. In China and Hong Kong hypertension is the most common cause, particularly in women, although coronary artery disease with diabetes is almost as common in men. ${ }^{5}$ In sub-Saharan Africa rheumatic heart disease is still common, especially in the young, but hypertension is the major cause in the large cities. ${ }^{8}$ In certain areas of Africa dilated cardiomyopathy, endomyocardial fibrosis, and peripartum heart failure (related to local heating customs in northern Nigeria ${ }^{9}$ ) are relatively common causes of hospital admission for heart failure. ${ }^{810}$ Interestingly hypertensive heart failure was the most common cause of sudden unexpected death in Nigeria, unlike in the west where coronary artery disease predominates. ${ }^{11}$ Chaga's disease is still an important and understated cause of heart failure in South America, but coronary artery disease is increasingly the cause in the majority of cases. ${ }^{4}$ The high prevalence of hypertension in Asia probably accounts for the high frequency (about $50 \%$ ) of heart failure with a relatively normal left ventricular ejection fraction, labelled as "diastolic heart failure".$^{12}$ However, the rise of coronary artery disease means it is likely to become the major cause in South Asia and China in the near future. In these two continents in which over half the world's population live it is estimated that death from coronary heart disease will rise by $115-127 \%$ for Indian and $81-110 \%$ for Chinese women and men, respectively, from the level in 1990 to $2020 .^{13}$ These estimates are based on demographic changes alone and do not take into account major increases in risk factors which are likely on present trends.

\section{TREATMENT}

Fortunately, for a previously dismal disease the treatment of heart failure has improved notably over the past 20 years. The most effective drug treatments (diuretics, angiotensin converting enzyme (ACE) inhibitors, $\beta$ blockers, and spironolactone) are relatively cheap and could be available to the majority in most areas of the world. The more expensive options such as surgery, transplantation, left ventricular assist devices, and biventricular pacing are clearly only going to be available for a minority even in the richest economies. The evidence that different ethnic groups have the same mortality benefit from these standard medications is slim, as few large scale trials have included regions outside Europe and the USA, although smaller clinical trials have confirmed the effectiveness of ACE inhibitors and $\beta$ blockers in Africa and Asia. ${ }^{14}{ }^{15}$ It is likely, given the fundamental nature of the derangements in heart failure, that blockade of neurohormonal activation will be equally effective universally although dosages may differ slightly.

As always the concluding plea is the same; the answer is better prevention, particularly in developing or newly industrialised countries where an epidemic of cardiovascular diseases and diabetes is now unfolding. Treatment of heart failure is still expensive and not entirely successful, and the impairment of quality of life for many elderly by heart failure symptoms is substantial. But there is ground for some optimism. A large body of studies now implicates a few well known risk factors as the major determinants of both coronary 
artery disease and hypertension in the majority of individuals, and these are mainly caused by the changes in lifestyle and diet that accompany economic growth and industrialisation. ${ }^{2}$ Although newer risk factors and genetic polymorphisms may generate some academic interest we know enough already to be able to prevent the majority of cardiovascular diseases. Neither coronary artery disease nor hypertension need to exist and their elimination would also remove the two most debilitating vascular diseases, namely heart failure and stroke. Perhaps our targets should now be not only the tobacco trade but also the fast food industry, both of which are making great inroads in many developing countries.

\section{Authors' affiliations}

J E Sanderson, Department of Medicine and Therapeutics, The Chinese University of Hong Kong, Prince of Wales Hospital, Hong Kong SAR T-f Tse, Past-President World Heart Federation, c/o Hong Kong College of Cardiology, Hong Kong SAR

\section{REFERENCES}

1 Chockalingam A, Balaguer-Vintro I. Impending global pandemic of cardiovascular diseases. Barcelona: World Heart Federation. Prous Science SA, 1999.

2 Yusuf S, Reddy S, Ounpuu S, et al. Global burden of cardiovascular diseases. Part I: general considerations, the epidemiologic transition, risk factors, and impact of urbanization. Circulation 2001;104:2746-53.

3 McMurray JJV, Stewart S. Epidemiology, aetiology, and prognosis of heart failure. Heart 2000;83:596-602.
4 Mendez GF, Cowie MR. The epidemiological features of heart failure in developing countries: a review of the literature. Int J Cardiol 2001;80:213-19.

5 Sanderson JE, Chan S, Chan WWM, et al. The aetiology of heart failure in the Chinese population of Hong Kong - a prospective study of 730 consecutive patients. Int J Cardiol 1995;51:29-35.

6 Oyoo GO, Ogola EN. Clinical and socio demographic aspects of congestive heart failure patients at Kenyatta National Hospital, Nairobi. East Afr Med J 1999;76:23-7.

7 Agarwal AK, Venugopalan P, de Bono D. Prevalence and aetiology of heart failure in an Arab population. Eur J Heart Failure 2001;3:301-5.

8 Amoah AGB, Kallen C. Aetiology of heart failure as seen from a national cardiac referral centre in Africa. Cardiology 2000;93:11-18.

9 Sanderson JE. Oedema and heart failure in the tropics. Lancet 1977; ii: $1159-61$.

10 Ford L, Abdullahi A, Anjorin Fl, et al. The outcome of peripartum cardiac failure in Zaria, Nigeria. QJM 1998;91:93-103.

11 Rotimi O, Ajayi AA, Odesanmi WO. Sudden unexpected death from cardiac causes in Nigerians: a review of 50 autopsied cases. Int J Cardiol 1998;63:111-15.

12 Yip GWK, Ho PPY, Woo KS, et al. Comparison of frequencies of left ventricular systolic and diastolic heart failure in Chinese living in Hong Kong. Am J Cardiol 1999;84:563-7.

13 Murray CJL, Lopez AD. The global burden of disease: a comprehensive assesment of mortality and disability from diseases, injuries, and risk factors in 1990 and projected to 2020. Boston: Harvard School of Public Health, 1996.

14 Ajayi AA, Balogun MO, Oyewo EA, et al. Enalapril in African patients with congestive heart failure. Br J Clin Pharm 1989;27:400-3

15 Sanderson JE, Chan SKW, Yip GWK, et al. Beta-blockade in heart failure: a comparison of carvedilol with metoprolol. J Am Coll Cardiol 1999;34:1522-8.

\section{IMAGES IN CARDIOLOGY}

An unusual case of chest murmur demonstrated with three dimensional volume rendering with 16 row multislice spiral computed tomography

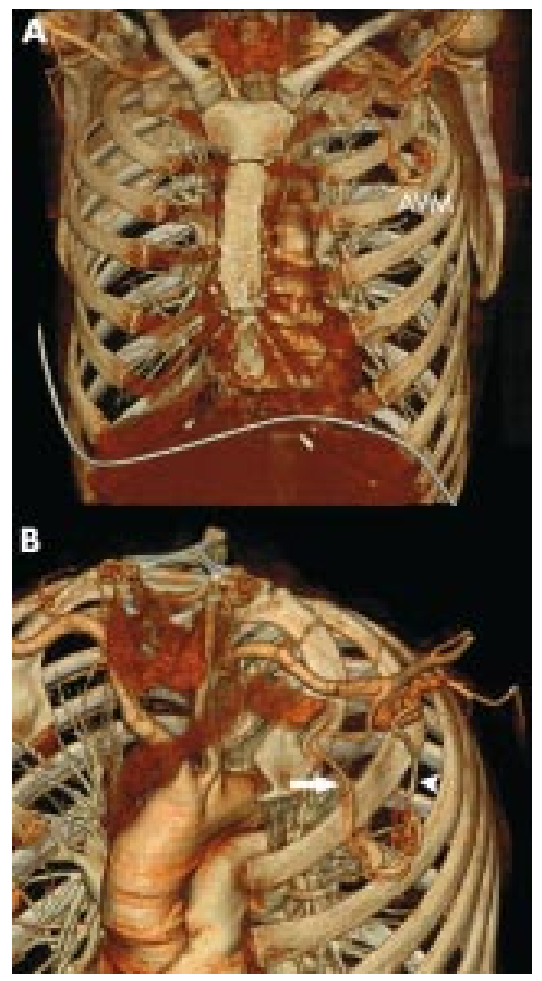

62 year old woman was admitted to our outpatient department because of an
unusual precordial murmur detected during a physical examination performed by her general practitioner after an upper airway infection.

Physical examination confirmed a continuous murmur (crescendo-decrescendo) maximal at the left second intercostal space along the midclavicular line. ECG sinus rhythm, chest $x$ ray, and transthoracic and transoesophageal ultrasounds were normal.

A contrast enhanced 16 slice spiral CT (MSCT) of the entire thorax (panels A and B) showed an arteriovenous malformation (AVM) originating from the left subclavian artery (panel $\mathrm{B}$, arrow) and draining into the subclavian vein (arrowhead). Three dimensional reconstruction with volume rendering shows the anatomy, location, and configuration of the vascular anomaly (panels A and B). The patient referred to a chest trauma more than 20 years ago with specific details. At three months follow up she was still asymptomatic.

The therapeutic decision was that only after symptoms or evidence of progression of the arteriovenous malformation should a percutaneous coiling procedure be performed. 